\title{
Transient estrogen exposure from birth affects uterine expression of developmental markers in neonatal gilts with lasting consequences in pregnant adults
}

\author{
Joseph C Chen, Anne A Wiley, Teh-Yuan Ho, Amy-Lynn Frankshun, Kristin M Hord, \\ Frank $\mathrm{F} \mathrm{Bartol}^{1}$ and Carol A Bagnell \\ Department of Animal Sciences, Rutgers, The State University of New Jersey, 84 Lipman Drive, New Brunswick, \\ New Jersey 08901, USA and ${ }^{1}$ Department of Anatomy, Physiology and Pharmacology, Cellular and Molecular \\ Biosciences Program, Auburn University, Auburn, Alabama 36849, USA
}

Correspondence should be addressed to C A Bagnell; Email: bagnell@aesop.rutgers.edu

\begin{abstract}
Disruption of estrogen-sensitive, estrogen receptor (ER)-dependent events during porcine uterine development between birth (postnatal day $=$ PND 0) and PND 14 affects patterns of uterine morphoregulatory gene expression in the neonate with lasting consequences for reproductive success. Uterine capacity for conceptus support is reduced in pregnant adult gilts exposed to estradiol valerate (EV) for 14 days from birth. Objectives here were to determine effects of EV exposure from birth through PND 13 on neonatal uterine and adult endometrial markers of growth, patterning, and remodeling. Targets included the relaxin receptor (RXFP1), estrogen receptor- $\alpha$ (ESR1) and vascular endothelial growth factor (VEGFA), morphoregulatory markers HOXA10 and WNT7A, and the matrix metalloproteinases (MMP)2 and MMP9. Gilts were treated daily with EV (50 $\mu \mathrm{g} / \mathrm{kg}$ body weight per day, i.m.) or corn oil vehicle from birth through PND 13. Uteri were obtained from neonates on PND 14 and from adults on pregnancy day 12 (PxD 12). In neonates, EV exposure from birth increased uterine RXFP1 gene expression, and both ESR1 and VEGFA proteins. At PXD 12, endometrial RXFP1 mRNA remained elevated, while ESR1 protein was reduced. Early EV treatment decreased neonatal uterine $W N T 7 A$, but increased HOXA10 expression. WNT7A expression was reduced in EV-treated adults. Transient EV exposure increased MMP9 transcripts at PND 14, whereas both latent and active MMP9 activity was increased due to early EV treatment in adults on PxD 12. Results support the hypothesis that transient, estrogen-induced disruption of porcine uterine development from birth alters early programming events that lead to functional consequences in the adult.

Reproduction (2010) 139 623-630
\end{abstract}

\section{Introduction}

Development of the uterus involves a series of morphogenetic and cytodifferentiative events that establish the framework for tissue function in adulthood. In the pig (Sus scrofa domesticus), uterine glands are absent at birth (postnatal day (PND) 0) and the uterus is estrogen receptor- $\alpha$ (ESR1) negative (Tarleton et al. 1998, Yan et al. 2006a). However, during the first 2 weeks of life, uterine glands differentiate and ESR 1 expression is evident in both nascent glandular epithelium and endometrial stroma (Tarleton et al. 1999). These events are ovary independent and estrogen-sensitive (Bartol et al. 1993). Disruption of estrogen-sensitive uterine developmental events by exposure of neonatal gilts to estrogen from birth can have both short- and long-term consequences.

Administration of estrogens to neonatal gilts from birth affects uterine growth and endometrial development acutely at both structural and biochemical levels.
Short-term exposure to estrogens from birth showed that uterine expression of the relaxin receptor (RXFP1), ESR1, and vascular endothelial growth factor (VEGFA) increased as early as PND 2 (Yan et al. 2008). Treatment with estradiol valerate (EV) for 2 weeks from birth increased uterine wet weight and advanced endometrial development by PND 14 as reflected by increased glandularity and premature development of endometrial folds (Tarleton et al. 1999). These effects were associated with changes in endometrial expression patterns for morphoregulatory genes in the WNT/HOXA family (Bartol et al. 2006).

In contrast, exposure of neonatal gilts to EV for 2 weeks from birth had anti-uterotropic effects in pregnant adult gilts. Neonatally estrogen-exposed adult gilts had smaller uteri at day 12 post estrus/mating. This effect was most pronounced in pregnant, as compared to cyclic, animals (Tarleton et al. 2003). Consistently, adult pregnant gilts that were exposed to EV neonatally 
displayed reduced uterine capacity on pregnancy day 45 (PxD 45) as reflected by reduced embryo survival (Bartol et al. 1993). Furthermore, early estrogen exposure did not affect ovulation rate or prevent conception, suggesting that the reduction in uterine capacity was the result of direct $\mathrm{EV}$ effects on neonatal uterine programming (Bartol et al. 1993, Tarleton et al. 2003).

Given that estrogen-induced disruption of neonatal uterine development has long-term consequences for uterine function and reproductive performance in the pig, it is reasonable to expect that associated changes in uterine expression of growth, patterning, and remodeling genes during neonatal life could mark and even mediate important developmental programming events. In this regard, both RXFP1 and ESR1 signaling systems have been implicated in an estrogen-sensitive, feed-forward system regulating uterine growth and endometrial development in the neonatal pig (Bartol et al. 2009). In addition, endometrial expression of patterning genes including WNT7A and HOXA10 is recognized to be developmentally regulated and estrogen sensitive (Bartol et al. 2006). Tissue remodeling matrix metalloproteinases (MMPs), specifically the gelatinases MMP2 and MMP9, have yet to be evaluated in terms of their potential involvement in neonatal porcine uterine developmental programming. However, it is known that these enzymes are responsible for the coordinated breakdown of the extracellular matrix (ECM) and basement membrane remodeling important for tissue expansion and that these MMPs increase during relaxin-induced uterine growth in prepubertal gilts (Lenhart et al. 2001).

In studies by Tarleton et al. (2003), EV was used as a tool with which to disrupt estrogen-sensitive developmental events in the neonate in order to identify longterm outcomes of such developmental disruption on uterine morphology, biochemistry, and functionality in cyclic adult and early pregnant gilts, in which effects were most pronounced. Objectives of the current study were to extend these observations by determining: 1) short-term effects of $E V$, administered daily from birth through PND 13, on molecular markers of uterine growth, patterning, and remodeling at PND 14; and 2) long-term effects of this neonatal estrogen exposure strategy on these markers of endometrial development in adults at PxD 12.

\section{Results}

\section{Neonatal uterine and adult endometrial mRNA expression}

Data for the relative expression of uterine developmental genes in the neonate at PND 14 and at PxD 12 as a result of neonatal EV treatment are presented in Figs 1 and 2 respectively. Neonatal exposure to EV for 2 weeks from birth increased $(P<0.05)$ uterine wet
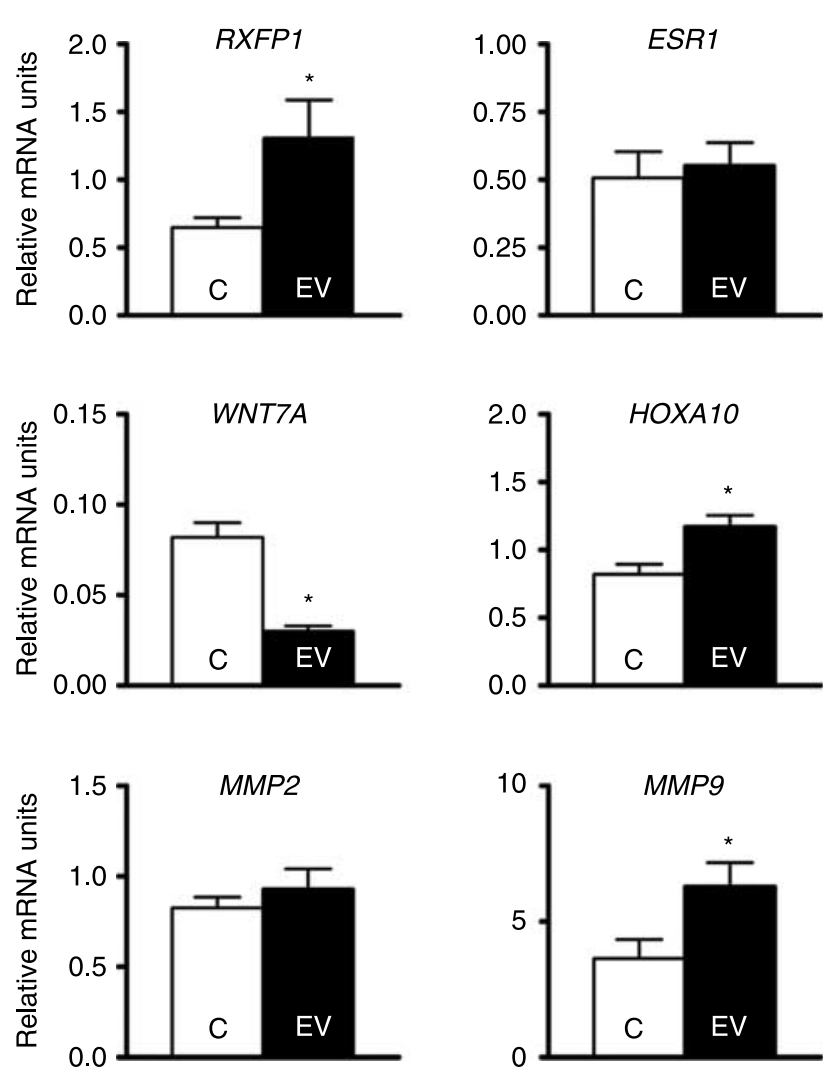

Figure $1 \mathrm{Effects}$ of $\mathrm{EV}$, administered from PND 0 to PND 13, on porcine uterine gene expression at PND 14. Transcripts for RXFP1, ESR1, WNT7A, HOXA10, MMP2, and MMP9 were quantified by qPCR and normalized to the expression of the housekeeping gene PPIA. Data are expressed as LSM \pm S.E.M. $N=4-6$ animals per group. ${ }^{*} P<0.05$.

weight (EV: $1.40 \pm 0.16 \mathrm{~g}$ ) compared to controls (C: $0.53 \pm 0.07 \mathrm{~g}$ ) on PND 14, similar to previously reported data (Tarleton et al. 1999). Two weeks of EV treatment from birth decreased $(P<0.05)$ porcine uterine WNT7A mRNA and increased $(P<0.05)$ RXFP1, HOXA10, and MMP9 transcripts at PND 14. However, there were no EV-induced changes in uterine ESR1 or MMP2 gene expression at PND 14. In adults at PxD 12, transient neonatal EV exposure decreased $(P<0.05)$ endometrial WNT7A mRNA and increased $(P<0.05)$ endometrial RXFP1 mRNA. Contrary to uterine PND 14 results, at PxD 12 there was a decrease $(P<0.05)$ in endometrial MMP9 transcripts in gilts transiently exposed to EV for 2 weeks from birth. There were no EV-induced changes in endometrial ESR1 or HOXA10 gene expression when measured at PXD 12.

\section{Neonatal uterine and adult endometrial ESR1 and VEGFA protein expression}

Results of immunoblot analysis of neonatal uterine and adult endometrial proteins are shown in Figs 3 and 4 respectively. EV treatment of neonatal gilts during the first 2 weeks of life increased $(P<0.05)$ the relative 

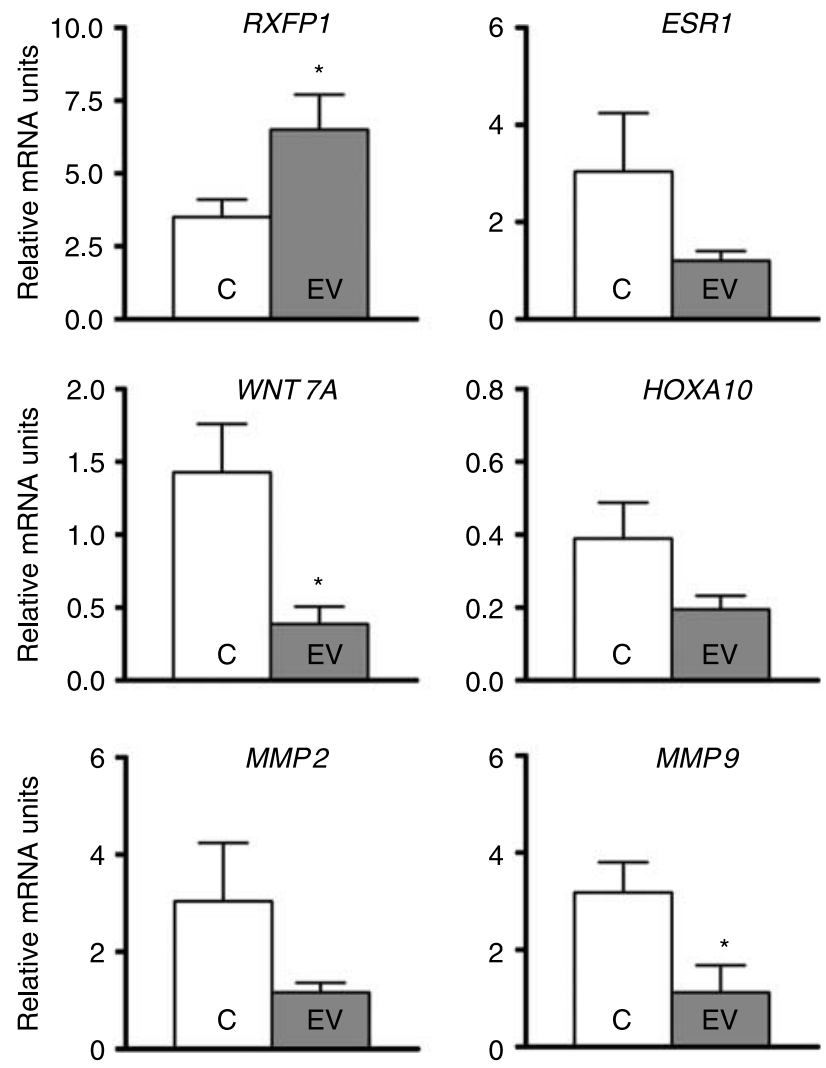

Figure 2 Effects of EV, administered from PND 0 to PND 13, on porcine endometrial levels of mRNA at PxD 12. Transcripts for RXFP1, ESR1, WNT7A, HOXA10, MMP2, and MMP9 were determined by qPCR and normalized to the expression of the housekeeping gene PPIA. Data are expressed as LSM \pm S.E.M. $N=4-6$ animals per group. ${ }^{*} P<0.05$.

abundance of a single $51 \mathrm{kDa}$ uterine protein band, corresponding to ESR1 (Fig. 3A), and a $46 \mathrm{kDa}$ band, corresponding to dimeric VEGFA protein (Fig. 3B) at PND 14. At PxD 12, transient exposure to EV for 2 weeks from birth decreased the relative abundance of endometrial ESR1 protein (Fig. 4A; $P<0.05$ ); however, there were no changes in VEGFA protein levels (Fig. 4B).

\section{Neonatal uterine and adult endometrial MMP2 and MMP9 gelatinase activity}

Gelatin zymography was used to identify the type and measure the abundance of gelatinases in neonatal uterine and adult endometrial tissues. Representative zymograms illustrating the gelatinolytic activity of neonatal uterine and adult endometrial protein extracts are shown (Fig. 5). Zymographic analysis of PND 14 uterine proteins revealed the presence of lysis bands at 72 and $66 \mathrm{kDa}$ (Fig. 5A), the reported sizes of pro-MMP2 and MMP2 respectively (Crabbe et al. 1993). However, densitometric analysis of these uterine MMP2 lysis bands indicated no effect of $\mathrm{EV}$ treatment for 2 weeks from birth in comparison to controls (data not shown). Zymographic analysis of PxD 12 adult endometrial proteins
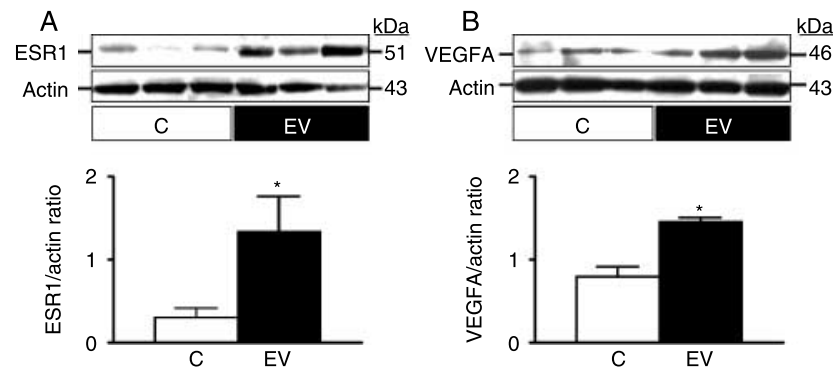

Figure 3 Effects of EV, administered from PND 0 to PND 13, on porcine uterine ESR1 (A) and VEGFA (B) proteins at PND 14. Representative immunoblots for each treatment group are shown. Both 51 and $46 \mathrm{kDa}$ immunoreactive bands for ESR1 and VEGFA respectively are indicated (A and B). An immunoreactive band for actin was detected at $43 \mathrm{kDa}$ and included as a loading reference. Densitometric data for the relative expression of ESR1 and VEGFA are expressed as LSM \pm S.E.M. $N=4-6$ animals per group. ${ }^{*} P<0.05$.

revealed the presence of lysis bands at 92 and $84 \mathrm{kDa}$ (Fig. 5B), the reported sizes of pro-MMP9 and MMP9 respectively (O'Connell et al. 1994). In addition, bands for pro-MMP2 and MMP2 were also detected (Fig. 5B). Densitometric analyses illustrate that neonatal EV treatment resulted in a decline in both endometrial pro-MMP9 and MMP9 gelatinase activity at PxD 12 (Fig. 5C; $P<0.05$ ). There were no differences in endometrial pro-MMP2 or MMP2 gelatinase activity at PxD 12 as a result of transient EV exposure for 2 weeks from birth.

\section{Discussion}

Studies in the pig show that there are dynamic changes in the morphology and molecular profile of the porcine uterus during the first 2 weeks of life (Bartol et al. 2006), and that exposure to estrogens during this period can compromise adult uterine function (Tarleton et al. 2003). Although developmentally disruptive effects of estrogen
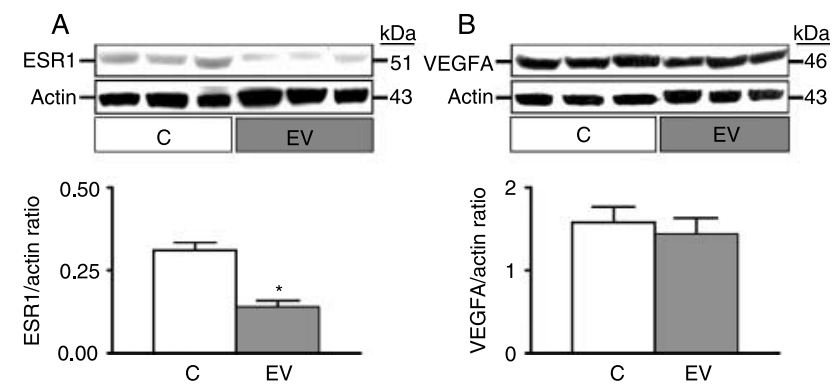

Figure 4 Effects of EV, administered from PND 0 to PND 13, on porcine uterine ESR1 (A) and VEGFA (B) proteins at PxD 12. Representative immunoblots for each treatment group are shown. Both 51 and $46 \mathrm{kDa}$ immunoreactive bands for ESR1 and VEGFA respectively are indicated (A and B). An immunoreactive band for actin was detected at $43 \mathrm{kDa}$ and included as a loading reference. Densitometric data for the relative expression of ESR1 and VEGFA are expressed as LSM \pm s.E.M. $N=4-6$ animals per group. ${ }^{*} P<0.05$. 
A

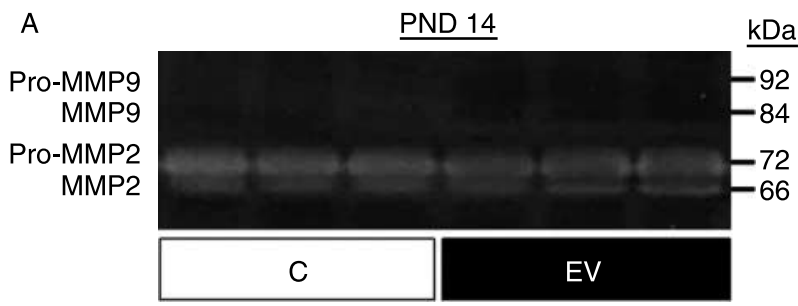

B

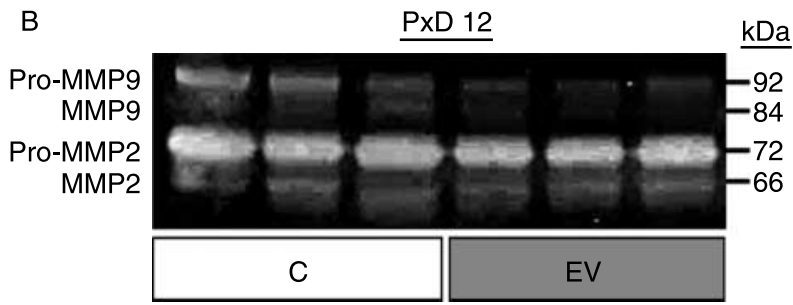

C

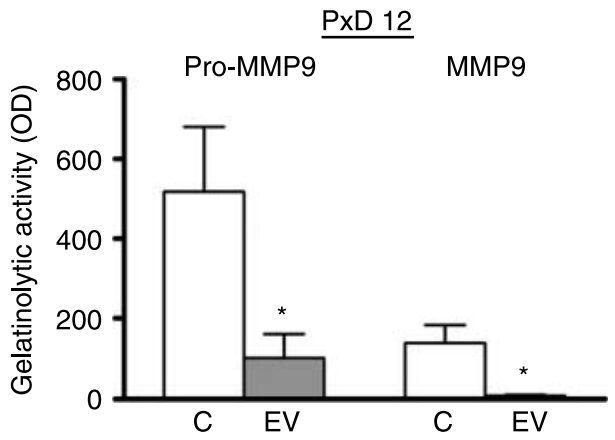

Figure 5 Effects of EV, administered from PND 0 to PND 13, on porcine uterine MMP9 and MMP2 gelatinase activity at PND 14 (A) and PxD 12 (B). Representative zymograms for each age and treatment group are shown. In the zymograms, clear zones against the dark background indicate gelatinolytic activity for pro-MMP9 (92 kDa), MMP9 (84 kDa), pro-MMP2 (72 kDa), and MMP2 (66 kDa). (C) Endometrial MMP9 gelatinolytic activity at PxD 12 was quantified by densitometry and graphed in OD units (LSM \pm S.E.M.; $N=4-6$ animals per group). ${ }^{*} P<0.05$.

exposure on the uterus have been documented, identifying the programming pathways that are altered by such exposures is an ongoing process. This study was designed to evaluate both short- and long-term programming effects of transient neonatal estrogen exposure on developmental markers of porcine uterine growth, patterning, and remodeling. Results show clearly that disruption of estrogen-sensitive early neonatal uterine developmental events affects the developmental trajectory and, ultimately, function of adult endometrium during the periattachment period of early pregnancy.

Porcine uterine ESR1 expression develops in an agedependent manner during the first 2 weeks of life (Yan et al. 2006a) and can be activated by exogenous estrogens as early as PND 2 (Yan et al. 2008). In the current study, transient neonatal EV exposure from birth increased uterine ESR1 protein at PND 14. These data support the histological evidence that estrogens increased ESR1positive uterine gland development during the first 2 weeks of life (Tarleton et al. 1999). The increase in uterine
ESR1 protein occurred concurrently with an increase in uterine VEGFA protein, a marker of estrogen action in immature rat (Hyder et al. 1996) and neonatal porcine (Yan et al. 2008) uterine tissues, as well as an indicator of tissue angiogenesis and growth. Moreover, present data indicate that, while short-term effects of neonatal estrogen exposure include increased ESR1 expression, long-term effects include a reduction in endometrial ESR1 protein. These relationships are complementary to data reported for uterine wet weight under identical treatment conditions indicating that while short-term effects of neonatal estrogen treatment were uterotropic, long-term effects, as assessed on PxD 12, were anti-uterotropic (Tarleton et al. 2003). Since endometrial ESR1 is required for both recognition of pregnancy in pigs (Bazer \& Thatcher 1977, Marengo et al. 1986) and the secretion of luminal proteins necessary for conceptus development (Flint et al. 1978, Roberts et al. 1993, Tarleton et al. 2003), decreases in uterine ESR1 expression induced by disruption of estrogen-sensitive developmental programming events can likely compromise endometrial capacity for support of the conceptus, as reflected by reduced embryonic survival (Bartol et al. 1993).

Relaxin, a milk-borne hormone, has been found in rodent, porcine, and human milk (Eddie et al. 1989, Steinetz et al. 1996, Yan et al. 2006b). Bioactive relaxin is present in porcine milk (Frankshun et al. 2009) and is detectable in the systemic circulation of newborn pigs only if they are allowed to nurse (Yan et al. 2006b, Frankshun et al. 2009). Relaxin increased uterine growth in neonatal gilts (Yan et al. 2006a) and stimulated expression of porcine uterine markers of growth and remodeling (Lenhart et al. 2001, Yan et al. 2008). Actions of relaxin are mediated via its receptor, RXFP1, a transmembrane protein belonging to the leucine-rich $G$ protein receptor family (Hsu et al. 2002). Results of the present study, indicating that exposure of gilts to EV for 2 weeks from birth increased uterine RXFP1 expression at PND 14, support previous studies that showed estrogens increased porcine uterine relaxin binding (Mercado-Simmen et al. 1982) as well as RXFP1 gene expression in the porcine reproductive tract (Yan et al. 2008) and in a human uterine fibroblast cell line (Maseelall et al. 2009). Importantly, data also show that the estrogen-induced increase in uterine RXFP1 expression observed on PND 14 is similar to that observed in adult endometrium obtained from neonatally EV-exposed gilts on PxD 12. These relationships could reflect neonatally programmed dysregulation of mechanisms governing dynamic adult endometrial RXFP1 expression at a time in early pregnancy when local estrogen signaling associated with maternal recognition of pregnancy in the pig is high (Tarleton et al. 2003). Data support the idea that uterine RXFP1 expression can be used as a marker of transient, neonatal estrogen exposure in both neonatal and adult periods. In human pregnancy, increased decidual relaxin 
(Bogic et al. 1997) and RXFP1 expression were linked to negative outcomes such as preterm delivery (Lowndes et al. 2006). Whether elevated endometrial RXFP1 in PxD 12 gilts treated neonatally with EV is associated with dysregulation of relaxin-mediated periattachment events and reduced uterine capacity is unknown.

Evidence from WNT7A and HOXA10 null mice demonstrated that these genes are not only responsible for organizationally critical uterine patterning and differentiation events (Benson et al. 1996, Miller \& Sassoon 1998), but that WNT7A is necessary to maintain the expression of uterine stromal patterning genes, including WNT4, WNT5A, and HOXA10 (Miller \& Sassoon 1998, Kitajewski \& Sassoon 2000). WNT7A expression is sensitive to estrogens, as murine models show that WNT7A expression in the uterus declines as estrus approaches and systemic estradiol increases (Kitajewski \& Sassoon 2000). This effect can be reproduced by exogenous exposure to estrogenic compounds (Miller et al. 1998). Thus, the estrogenregulated decline in WNT7A expression is postulated to be essential to insure up-regulation of patterning genes in the WNT/HOXA axis, including HOXA10, expression of which increases in response to estrogens (Taylor et al. 1998, Block et al. 2000, Taylor 2000). Exposure of fetal mice to estrogens resulted in uterine structural and functional irregularities that closely mimicked those observed in both WNT7A and HOXA10 knockout mice (Satokata et al. 1995, Miller \& Sassoon 1998), including homeosis of the reproductive tract and sterility (Miller et al. 1998, Block et al. 2000). Data reported here for uterine WNT7A and HOXA10 expression at PND 14 confirm and extend previous observations involving qualitative in situ hybridization analysis of the neonatal porcine uterus (Bartol et al. 2006). In that report, both WNT7A and HOXA10 mRNA developed in a timedependent manner from birth to PND 14, and treatment with EV for 14 days from birth reduced WNT7A expression in luminal epithelium and increased HOXA10 expression in endometrial stroma on PND 14. Again, estrogen-induced disruption of the neonatal developmental program documented here was also marked by reduced endometrial WNT7A expression on PxD 12 in neonatally estrogen-exposed pregnant adults. Present data indicating that disruption of the neonatal porcine uterine WNT/HOXA expression axis has longterm implications for endometrial function in the pig should not be surprising in light of similar data reported for the mouse.

While studies show that exposure of the prepubertal pig to relaxin increased uterine secretion of gelatinases, MMP2 and MMP9 (Lenhart et al. 2001), relatively little is known about the expression and activity of gelatinases in the neonatal porcine uterus. Even less is known about the functional consequences of dysregulated neonatal uterine MMP expression in the pig. MMPs are a family of proteinases that are responsible for degrading the ECM to facilitate tissue growth and remodeling (Hulboy et al. 1997, Visse \& Nagase 2003). Gelatinolytic degradation of the basement membrane and the ECM allows for tissue expansion (Hulboy et al. 1997, Masson et al. 2005) and release of growth factors present in the ECM (Vukicevic et al. 1992). Both MMP2 and MMP9 have been detected in reproductive tissues and implicated as major players in uterine remodeling in several mammalian species, including mice (Hu et al. 2004), pigs (Lenhart et al. 2001), and humans (Martelli et al. 1993). Moreover, there is evidence that MMP2 and MMP9 are regulated, in part, by estrogens. In rat endometrial tumor cells, estrogen exposure induced MMP2 transcripts (Tushaus et al. 2003) and, in mouse uterus, MMP9 activity increased with estrogen exposure (Zhang et al. 2007). Here, data indicate that neonatal EV treatment increased uterine MMP9 expression at PND 14. Conversely, in the adult endometrium at PxD 12, neonatal EV treatment reduced MMP9 expression, with corresponding decreases observed for both latent and active MMP9 protein activity. The expression of MMP9 transcripts and related protein activity at neonatal and adult time points complements changes associated with uterine weight as a result of transient neonatal EV exposure (Tarleton et al. 1999, 2003). Since tissue growth relies on the coordinated activity of MMPs, a reduction in MMP9 activity at PxD 12 may have contributed to the reduction in uterine size in adult gilts exposed neonatally to EV. These data provide evidence that estrogen-sensitive developmental programming of porcine uterine tissues between birth and PND 14 may also involve MMP9 as an important regulatory element.

Given that neonatal EV treatment did not affect ovulation rates or the ability of gilts to cycle or conceive (Bartol et al. 1993, Tarleton et al. 2003), it can be inferred that embryonic losses associated with such neonatal estrogen exposure were due to the direct effects of $\mathrm{EV}$ on uterine programming events. Collectively, data reinforce the idea that estrogen-sensitive markers of neonatal porcine uterine growth, patterning, and remodeling can be altered during the first 2 weeks of neonatal life with consequences in adults. These markers of uterine development should be included as elements of the organizational palette of factors that define the porcine uterine developmental program and determine the developmental trajectory of uterine tissues, including the endometrium. Temporospatial uterine expression patterns observed for such genes shortly after birth can define the potential for developmental success and determine the functional capacity of adult uterine tissues (Bartol et al. 2006). Identification of the complete array and role of factors that define the porcine uterine developmental program and determine the developmental trajectory of these tissues will provide an important insight into mechanisms regulating reproductive efficiency and performance. 


\section{Materials and Methods}

\section{Materials}

TRI Reagent was obtained from Sigma-Aldrich. RNeasy Mini kits and RNase-Free DNase Sets were obtained from Qiagen Inc. SuperScript III First-Strand Synthesis System for RT-PCR was from Invitrogen. SYBR Green PCR Master Mix was purchased from Applied Biosystems (Foster City, CA, USA). Primers were synthesized by Sigma Genosys. Detergent-compatible protein assay kits (DC Protein Assay) were purchased from Bio-Rad Laboratories. Mouse antihuman ESR1 (Ab-15) mAb was from NeoMarkers, Inc. (Fremont, CA, USA). Rabbit anti-human VEGFA (A-20-G) and goat anti-human actin (sc-1615) polyclonal antibodies were from Santa Cruz Biotechnology, Inc. (Santa Cruz, CA, USA). HRP-conjugated anti-mouse and anti-goat secondary antibodies were purchased from Zymed (Carlsbad, CA, USA). Nitrocellulose membranes were obtained from Bio-Rad Laboratories. The Renaissance Western Blot Chemiluminescence Reagent Plus kits were acquired from Perkin Elmer Life Sciences (Waltham, MA, USA). XOmatic films were purchased from American Imaging (South Plainfield, NJ, USA). Zymogram gels and buffers were purchased from Invitrogen. EV and other chemicals were purchased from Sigma-Aldrich and Invitrogen, unless otherwise specified.

\section{Experimental design}

At birth, crossbred gilts (Sus scrofa domesticus) were assigned randomly to one of two neonatal treatment groups $(n=4-6$ gilts/group). Care was taken to ensure that treatments were balanced for potential effects of litter and sows were nursing litters of similar size. Neonatal treatments, administered as daily injections from birth (PND 0) through PND 13, were either corn oil vehicle (C; $50 \mu \mathrm{l} / \mathrm{kg}$ body weight (BW) per day, i.m.) or EV (50 $\mu \mathrm{g} / \mathrm{kg}$ BW per day, i.m.). The dosage, timing, and route of EV administration were based on previous studies (Tarleton et al. 2003). In study 1, neonatal uterine tissues from gilts were collected on PND 14 from both EV- and vehicletreated gilts. In study 2, neonatally treated gilts were allowed to reach puberty as evidenced by display of at least two consecutive estrous cycles of normal length. At the second estrus, gilts from each treatment group were bred by natural service at first estrus and again $24 \mathrm{~h}$ later. Endometrial tissues were collected from the adults at PxD 12.

\section{Tissue collection, RNA isolation, and cDNA generation}

In study 1, neonatal uterine tissues were removed, trimmed of fat and associated tissues and ligaments, and wet weights were recorded. In study 2, pregnant gilts were hysterectomized on day 12 post mating. Each uterine horn was flushed to confirm the presence of conceptus tissues and pregnancy as previously described (Tarleton et al. 2003). Uteri were opened along their mesometrial border, and the endometrium was harvested using a scalpel and forceps (Tarleton et al. 2003). Uterine tissues from PND 14 and endometrium from PxD 12 gilts were frozen using liquid nitrogen and stored at $-80^{\circ} \mathrm{C}$. Animals in study 1 were obtained from the Swine Unit of the New Jersey Agricultural Experiment Station, Rutgers University. Animals in study 2 were obtained from the Auburn University Swine Research and Education Center. The tissues used in study 2 were the same endometrial tissues used in the 2003 Tarleton study. All procedures involving animals were reviewed and approved by relevant Institutional Animal Care and Use Committees and were conducted in accordance with the Guide for the Care and Use of Agricultural Animals in Agricultural Research and Teaching (1999; Federation of Animal Science Society, Savoy, IL, USA).

Total RNA was isolated from 30 to $50 \mathrm{mg}$ tissue for each sample using TRI Reagent and the RNeasy Mini kit. Traces of DNA were removed using the RNase-Free DNase Set. RNA concentration and purity were evaluated by spectrophotometry. RNA integrity was checked by agarose gel electrophoresis and ethidium bromide staining to visualize sharp, clear $28 \mathrm{~S}$ and $18 \mathrm{~S}$ rRNA bands. RT was performed with 500 ng total RNA per sample using the PTC-200 Peltier Thermal Cycler (Bio-Rad Laboratories Inc.) and SuperScript III First-Strand Synthesis System for RT-PCR. All procedures were carried out following the manufacturers' instructions.

\section{Real-time RT-PCR}

Real-time RT-PCR (qPCR) was performed using an Applied Biosystems Gene Amp 7000 Sequence Detection System (Applied Biosystems) with the SYBR Green method following the universal thermal cycling parameters indicated by the manufacturer. Primers for qPCR were designed using Primer Express Software (Applied Biosystems) and synthesized by Sigma Genosys. All primer sequences were directed to the porcine genome (Table 1). To ensure specific amplification, controls including water only, no primers, and no template were included in the assay. The quality of the primers was

Table 1 Porcine primer accession numbers, sequences, and amplicon sizes for targeted uterine developmental genes.

\begin{tabular}{lclc}
\hline Gene & Accession number & Forward primer & Reverse primer \\
\hline WNT7A & CA997684 & CACCACCAAGACCTGCTGG & TCCTTGAGCACGTAGCCCA \\
HOXA10 & AF281156 & CGGCCGGAAGAAGCA & AGAAACTCCTTCTCCAGCTCCA \\
ESR1 & AF035775 & AGGGAGAGGAGTTTGTGTG & TCTCCAGCAGCAGGTCATAG \\
RXFP1 & CA994862 & GCATCACTTTGAGGCAGAGACA & CCTCGGCAAAGACATTGCAT \\
MMP2 & NM214192 & GAGCACCATCGAGACCATGA & TTGTAATTGGCCACGTCGG \\
MMP9 & DQ132879 & TGGATCCAAAACTACTCGGAAGAC & CGGACAAAGGCGTCG \\
PPIA & AU058466 & TTATAAAGGTTCCTGCTTTCACAGAA & TGCCATTATGGCGTGTGAAG \\
\hline
\end{tabular}


evaluated by amplifying serial dilutions of the cDNA template. In addition, dissociation curves for each set of primers were checked to ensure no amplicon-independent amplification (i.e. primer-dimer). PCR amplification products were analyzed by agarose gel electrophoresis to further confirm the absence of non-specific amplification. Data were analyzed using the relative standard curve method for quantitation of gene expression as described by Applied Biosystems (ABI User Bulletin 2, 2001). Standard curves were generated for each gene using two-fold dilutions of cDNA from PND 14 uterus (study 1) or PxD 12 endometrium (study 2; Larionov et al. 2005). Target gene expression was normalized to the expression of porcine cyclophilin (PPIA), and data from qPCR analyses are presented as relative mRNA units.

\section{Protein extraction and evaluation of ESR1 and VEGFA expression}

Uterine and endometrial tissue proteins were quantified using procedures described previously (Yan et al. 2008). Tissues (20-50 mg) were homogenized in $200 \mu$ l lysis buffer (1\% Triton X-100, 10\% glycerol, 150 mM Tris- $\mathrm{HCl}, 300 \mathrm{mM}$ $\mathrm{NaCl}$, and $1 \mathrm{mM} \mathrm{MgCl}_{2}, \mathrm{pH}$ 7.5). Samples were then sonicated and centrifuged $\left(12000 \mathrm{~g}, 4^{\circ} \mathrm{C}\right)$ for $30 \mathrm{~min}$, and the protein supernatant was removed and stored at $-20^{\circ} \mathrm{C}$. Protein concentration was measured using the DC Protein Assay kit (Bio-Rad). To document ESR1 and VEGFA protein expression, uterine proteins $(30 \mu \mathrm{g})$ were resolved on $12.0 \%$ total monomer, Bis-Tris-HCl-buffered polyacrylamide gels under reducing conditions and transferred onto nitrocellulose membranes. After blocking in $10.0 \%$ non-fat dry milk in Trisbuffered saline containing Tween-20 (TBST; $25 \mathrm{mM}$ Tris $(\mathrm{pH}$ 7.5), $0.14 \mathrm{mM} \mathrm{NaCl}, 3 \mathrm{mM} \mathrm{KCl}$, and $0.05 \%$ Tween-20), membranes were probed with either mouse anti-human ESR1 antibody $(1: 100)$ or rabbit anti-human VEGFA antibody (1:1000) overnight at $4{ }^{\circ} \mathrm{C}$. After washing with TBST, blots were incubated with either HRP-conjugated anti-mouse secondary antibody $(1: 1000)$ or anti-rabbit secondary antibody (1:1000) for $1 \mathrm{~h}$ at room temperature. Bound antibodies were detected by ECL. Protein loading was monitored using actin as a reference. Chemiluminescence signals were quantified densitometrically from film using Scion Image for Windows (Scion Corporation, Frederick, MD, USA).

\section{Gelatin zymography}

Zymography was performed as described previously (Ho et al. 2007). Briefly, samples (30 $\mu$ g protein) were mixed with equal amounts of SDS sample buffer and loaded under nondenaturing, non-reducing conditions using precast polyacrylamide zymogram gels supplemented with $1 \%$ gelatin as the proteinase substrate. Following electrophoresis, gels were washed in renaturing buffer for $30 \mathrm{~min}$ to remove the SDS and then incubated in developing buffer overnight at $37^{\circ} \mathrm{C}$. Gels were stained with $0.1 \%$ Coomassie brilliant blue R 250 . Human MMP2 and MMP9 enzyme standards were used as positive controls. Gelatinolytic activity of uterine and endometrial MMP2 and MMP9 was quantified densitometrically using Scion Image for Windows.

\section{Statistical analyses}

All data were subjected to analyses of variance. For relative gene and protein expression data (generated by densitometry of immunoblots), statistical models accounted for variation due to the main effects of treatment. Neonatal treatment effects on uterine (PND 14) and endometrial (PxD 12) responses were evaluated on a within-day basis only. Error terms were identified based upon the expectations of the mean squares for error, and data were expressed as least-square means with S.E.M.

\section{Declaration of interest}

The authors declare that there is no conflict of interest that could be perceived as prejudicing the impartiality of the research reported.

\section{Funding}

This work was supported by USDA-NRI-99-35203-7812, 2003-35203-13572, 2007-35203-18098, NSF EPS-0447675 and the NJ and AL Agricultural Experiment Stations.

\section{Acknowledgements}

The authors would like to thank Dr Becky J Tarleton Muir, Mr Brian Anderson and the staffs of the Auburn University and Rutgers University Animal Care Programs for their assistance in these studies.

\section{References}

Bartol FF, Wiley AA, Spencer TE, Vallet JL \& Christenson RK 1993 Early uterine development in pigs. Journal of Reproduction and Fertility Supplement 48 99-116.

Bartol FF, Wiley AA \& Bagnell CA 2006 Uterine development and endometrial programming. Society of Reproduction and Fertility Supplement 62 113-130.

Bartol FF, Wiley AA \& Bagnell CA 2009 Relaxin and maternal lactocrine programming of neonatal uterine development. Annals of the New York Academy of Sciences 1160 158-163.

Bazer FW \& Thatcher WW 1977 Theory of maternal recognition of pregnancy in swine based on estrogen controlled endocrine versus exocrine secretion of prostaglandin F2alpha by the uterine endometrium. Prostaglandins 14 397-400.

Benson GV, Lim H, Paria BC, Satokata I, Dey SK \& Maas RL 1996 Mechanisms of reduced fertility in Hoxa-10 mutant mice: uterine homeosis and loss of maternal Hoxa-10 expression. Development 122 2687-2696.

Block K, Kardana A, Igarashi P \& Taylor HS 2000 In utero diethylstilbestrol (DES) exposure alters Hox gene expression in the developing mullerian system. FASEB Journal 14 1101-1108.

Bogic LV, Yamamoto SY, Millar LK \& Bryant-Greenwood GD 1997 Developmental regulation of the human relaxin genes in the decidua and placenta: overexpression in the preterm premature rupture of the fetal membranes. Biology of Reproduction 57 908-920.

Crabbe T, Ioannou C \& Docherty AJ 1993 Human progelatinase A can be activated by autolysis at a rate that is concentration-dependent and enhanced by heparin bound to the C-terminal domain. European Journal of Biochemistry 218 431-438. 
Eddie LW, Sutton B, Fitzgerald S, Bell RJ, Johnston PD \& Tregear GW 1989 Relaxin in paired samples of serum and milk from women after term and preterm delivery. American Journal of Obstetrics and Gynecology 161 970-973.

Flint AP, Burton RD, Gadsby JE, Saunders PT \& Heap RB 1978 Blastocyst oestrogen synthesis and the maternal recognition of pregnancy. Ciba Foundation Symposium 64 209-238.

Frankshun AL, Ho TY, Steinetz BG, Bartol FF \& Bagnell CA 2009 Biological activity of relaxin in porcine milk. Annals of the New York Academy of Sciences 1160 164-168.

Ho TY, Yan W \& Bagnell CA 2007 Relaxin-induced matrix metalloproteinase-9 expression is associated with activation of the NF-kappaB pathway in human THP-1 cells. Journal of Leukocyte Biology 81 1303-1310.

Hsu SY, Nakabayashi K, Nishi S, Kumagai J, Kudo M, Sherwood OD \& Hsueh AJ 2002 Activation of orphan receptors by the hormone relaxin. Science 295 671-674.

Hu J, Zhang X, Nothnick WB \& Spencer TE 2004 Matrix metalloproteinases and their tissue inhibitors in the developing neonatal mouse uterus. Biology of Reproduction 71 1598-1604.

Hulboy DL, Rudolph LA \& Matrisian LM 1997 Matrix metalloproteinases as mediators of reproductive function. Molecular Human Reproduction 3 $27-45$.

Hyder SM, Stancel GM, Chiappetta C, Murthy L, Boettger-Tong HL \& Makela S 1996 Uterine expression of vascular endothelial growth factor is increased by estradiol and tamoxifen. Cancer Research 56 3954-3960.

Kitajewski J \& Sassoon D 2000 The emergence of molecular gynecology: homeobox and Wnt genes in the female reproductive tract. BioEssays 22 902-910.

Larionov A, Krause A \& Miller W 2005 A standard curve based method for relative real time PCR data processing. BMC Bioinformatics 662.

Lenhart JA, Ryan PL, Ohleth KM, Palmer SS \& Bagnell CA 2001 Relaxin increases secretion of matrix metalloproteinase-2 and matrix metalloproteinase-9 during uterine and cervical growth and remodeling in the pig. Endocrinology 142 3941-3949.

Lowndes K, Amano A, Yamamoto SY \& Bryant-Greenwood GD 2006 The human relaxin receptor (LGR7): expression in the fetal membranes and placenta. Placenta 27 610-618.

Marengo SR, Bazer FW, Thatcher WW, Wilcox CJ \& Wetteman RP 1986 Prostaglandin F2 alpha as the luteolysin in swine: VI. Hormonal regulation of the movement of exogenous PGF2 alpha from the uterine lumen into the vasculature. Biology of Reproduction 34 284-292.

Martelli M, Campana A \& Bischof P 1993 Secretion of matrix metalloproteinases by human endometrial cells in vitro. Journal of Reproduction and Fertility 98 67-76.

Maseelall P, Gardner J, Wojtczuk A, Weiss G \& Goldsmith LT 2009 Relaxin receptor LGR7 (RXFP1) is regulated by estrogen. Annals of the New York Academy of Sciences 1160 91-92.

Masson V, de la Ballina LR, Munaut C, Wielockx B, Jost M, Maillard C, Blacher S, Bajou K, Itoh T, Itohara S et al. 2005 Contribution of host MMP-2 and MMP-9 to promote tumor vascularization and invasion of malignant keratinocytes. FASEB Journal 19 234-236.

Mercado-Simmen RC, Goodwin B, Ueno MS, Yamamoto SY \& BryantGreenwood GD 1982 Relaxin receptors in the myometrium and cervix of the pig. Biology of Reproduction 26 120-128.

Miller C \& Sassoon DA 1998 Wnt-7a maintains appropriate uterine patterning during the development of the mouse female reproductive tract. Development 125 3201-3211.

Miller C, Degenhardt K \& Sassoon DA 1998 Fetal exposure to DES results in de-regulation of Wnt7a during uterine morphogenesis. Nature Genetics 20 228-230.
O'Connel JP, Willenbrock F, Docherty AJ, Eaton D \& Murphy G 1994 Analysis of the role of the $\mathrm{COOH}$-terminal domain in the activation, proteolytic activity, and tissue inhibitor of metalloproteinase interactions of gelatinase B. Journal of Biological Chemistry 269 14967-14973.

Roberts RM, Xie S \& Trout WE 1993 Embryo-uterine interactions in pigs during week 2 of pregnancy. Journal of Reproduction and Fertility Supplement 48 171-186.

Satokata I, Benson G \& Maas R 1995 Sexually dimorphic sterility phenotypes in Hoxa10-deficient mice. Nature 374 460-463.

Steinetz BG, Bullesbach EE, Goldsmith LT, Schwabe C \& Lust G 1996 Use of synthetic canine relaxin to develop a rapid homologous radioimmunoassay. Biology of Reproduction 54 1252-1260.

Tarleton BJ, Wiley AA, Spencer TE, Moss AG \& Bartol FF 1998 Ovaryindependent estrogen receptor expression in neonatal porcine endometrium. Biology of Reproduction 58 1009-1019.

Tarleton BJ, Wiley AA \& Bartol FF 1999 Endometrial development and adenogenesis in the neonatal pig: effects of estradiol valerate and the antiestrogen ICI 182,780. Biology of Reproduction 61 253-263.

Tarleton BJ, Braden TD, Wiley AA \& Bartol FF 2003 Estrogen-induced disruption of neonatal porcine uterine development alters adult uterine function. Biology of Reproduction 68 1387-1393.

Taylor HS 2000 The role of HOX genes in the development and function of the female reproductive tract. Seminars in Reproductive Medicine 18 81-89.

Taylor HS, Arici A, Olive D \& Igarashi P 1998 HOXA10 is expressed in response to sex steroids at the time of implantation in the human endometrium. Journal of Clinical Investigation 101 1379-1384.

Tushaus L, Hopert AC, Strunck E, Schubert C, Wunsche W \& Vollmer G 2003 Estrogenic and antiestrogenic regulation of MMP-2 and MMP-13 mRNA in RUCA-I endometrial tumor cells in vitro and in vivo. Cancer Letters 198 99-106.

Visse R \& Nagase H 2003 Matrix metalloproteinases and tissue inhibitors of metalloproteinases: structure, function, and biochemistry. Circulation Research 92 827-839.

Vukicevic S, Kleinman HK, Luyten FP, Roberts AB, Roche NS \& Reddi AH 1992 Identification of multiple active growth factors in basement membrane matrigel suggests caution in interpretation of cellular activity related to extracellular matrix components. Experimental Cell Research 202 1-8.

Yan W, Ryan PL, Bartol FF \& Bagnell CA 2006a Uterotrophic effects of relaxin related to age and estrogen receptor activation in neonatal pigs. Reproduction 131 943-950.

Yan W, Wiley AA, Bathgate RA, Frankshun AL, Lasano S, Crean BD, Steinetz BG, Bagnell CA \& Bartol FF 2006b Expression of LGR7 and LGR8 by neonatal porcine uterine tissues and transmission of milk-borne relaxin into the neonatal circulation by suckling. Endocrinology 147 4303-4310.

Yan W, Chen J, Wiley AA, Crean-Harris BD, Bartol FF \& Bagnell CA 2008 Relaxin (RLX) and estrogen affect estrogen receptor- $\alpha$, vascular endothelial growth factor and RLX receptor expression in the neonatal porcine uterus and cervix. Reproduction 135 705-712.

Zhang X, Christenson LK \& Nothnick WB 2007 Regulation of MMP-9 expression and activity in the mouse uterus by estrogen. Molecular Reproduction and Development 74 321-331.

Received 14 October 2009

First decision 3 November 2009

Accepted 23 December 2009 